\title{
An exact approach for the grocery delivery problem in urban areas
}

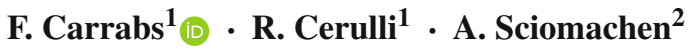

(C) Springer-Verlag Berlin Heidelberg 2016

\begin{abstract}
In this paper, we face the problem of delivering a given amount of goods in urban areas in a business-toconsumer (B2C) electronic commerce (EC) environment. This problem can be considered as a particular case of vehicle routing problem. As a novel issue, here we have to determine the fleet of no homogeneous vehicles to be used for satisfying the demands of clients coming from grocery echannels, and their related itineraries, given the traveling limits imposed by the urban government; in fact, commercial vehicles are not allowed to go everywhere and can travel only in restricted daily time windows, according to their pollution emissions. We have to minimize the overall distribution costs, taking into account traveling components and setup ones, together with operative aspects and environmental issues; customer requirements, vehicle capacity and daily shift constraints have to be satisfied too. We outline the main characteristics of the problem in a B2C EC environment and propose a mixed integer linear programming model to solve this NP-hard problem. Computational results of test bed cases related to different sized transportation networks and delivery demands are presented and analyzed with respect to the fleet of vehicles chosen for satisfying the customer demand
\end{abstract}

Communicated by V. Loia.

F. Carrabs

fcarrabs@unisa.it

R. Cerulli

raffaele@unisa.it

A. Sciomachen

sciomach@economia.unige.it

1 Department of Mathematics, University of Salerno, Via Giovanni Paolo II 132, 84084 Fisciano, SA, Italy

2 Department of Economics and Business Studies, University of Genoa, Via Vivaldi 5, 16125 Genoa, Italy and the street traffic limitations. Then, a realistic case study derived from the e-distribution channel of a grocery company of Genoa, Italy, is reported. Considerations about CPU time and optimality gap are also given with the idea of making the proposed model effectively used and solved with any commercial software.

Keywords Distribution network models - E-channel grocery delivery $\cdot$ Vehicle routing $\cdot$ Mixed integer linear programming models

\section{Introduction and problem definition}

Electronic commerce (EC) has seen tremendous growth rates in the recent years. From an operation management point of view, e-fulfillment, that is delivering physical goods to customers, is commonly cited as one of the most expensive and critical operations of EC. In order to face the complexities that companies have to manage regarding the underlying distribution processes, some sustainable models of e-commerce have recently started to emerge. The book edited by SimchiLevi et al. (2004) gives a detailed overview of related research areas. A survey of recent research on the impact of e-business on supply chain management is presented in Johnson and Whang (2002). Agatz et al. (2008) provide a review of valuable operations research models in connection to e-channel distribution problems; many are the variables involved in these distribution problems, depending on the objectives of the company. In particular, the location and type of the distribution centers, together with the number and type of vehicles used, distinguish the e-commerce distribution strategy of a company (Blumenfeld et al. 1985; De Koster 2002). The ability to adapt to late customer order impacts on the distribution strategy too. Note that the demand varies with time, space, 
and consumer socioeconomic characteristics. A mathematical programming model for determining how to optimize a delivery service strategy for EC in terms of service cycle is presented in $\mathrm{Hsu}$ and $\mathrm{Li}$ (2006); the authors consider time-dependent consumer demand and demand-supply interaction. Swaminathan and Tayur (2003) emphasize the supply chain aspects that increased their importance in EC and those that are new issues. In particular, several authors address the issue of choosing an appropriate delivery service level, in terms of time windows and lead times in EC (Punakivi and Saranen 2001; Hsu and Li 2006).

Concerning the grocery delivery, where low-value items are involved, transportation costs are the determinant key of the business viability; in fact, it is difficult to handle efficiently small transaction sized of individual customer orders. Further, as a distinctive factor of B2C in e-channel distribution, routing schedules have to be planned quite frequently (usually at least twice a day) since orders are small in size, instantaneous, ever changing, and placed by numerous consumers (Du et al. 2005). It is well known that the average logistics cost per item decreases with increasing delivery size; therefore, a larger quantity of goods can imply longer delivery cycles, which also results in an increased delay in receiving ordered goods. Consequently, to attend home delivery, both the company and its customers need to agree on a delivery time window. The length of this window and its daily timing are important aspects of the customer's perceived service; on the other side, many are the traffic restrictions concerning the daily delivery of goods in urban areas. For instances, in Italy the time windows allowed for the commercial traffic circulation in the main cities are limited to different time slots, also depending on their class of weight and pollution emission of the vehicles. An example of such time and street limits for the vehicles is reported in Table 1, where three classes of weight $\left(w_{1}, w_{2}\right.$ and $\left.w_{3}\right)$ and four types of emission $\left(e_{1}, e_{2}, e_{3}\right.$ and $\left.e_{4}\right)$ of the vehicles are considered.

Note that the above limitations impact on the delivery lead time and in turn on the seller's delivery costs.

The whole $\mathrm{B} 2 \mathrm{C}$ delivery process in the grocery market thus implies a trade-off between logistic costs and service level, expressed in terms of satisfaction of customer needs (Boyer et al. 2003). The problem is hence to find a distribution strategy that can satisfy both the capacity and time window constraints in a timely manner.

Having in mind the above-mentioned characteristics of e-channel B2C grocery delivery, in this paper we face the physical distribution processes in $\mathrm{B} 2 \mathrm{C}$ e-commerce concerning grocery delivery in urban and metropolitan areas. In particular, we face this problem by introducing a new variant of the classical VRP problem, named Street Constraints Vehicle Routing Problem (SCVRP); more precisely, we have to determine the fleet of no homogeneous vehicles, consisting of trucks, wagons, vans, and pickups, to be used for the delivery of a given amount of goods ordered via e-channels, taking into a proper account the government traffic restrictions of the urban area under study. In this direction, a number of papers on B2C urban distribution have been recently published by operations researchers and practitioners with the aim of providing advances for the development of a sustainable city logistics (see, e.g., Crainic et al. 2009; Qureshi et al. 2009; Cattaruzza et al. 2015). Kopfer et al. (2014) faced the problem of reducing the $\mathrm{CO} 2$ emissions caused by transportation; they embedded into Dantzig's classical vehicle routing model the option of choosing vehicles of different size for route fulfillment. The results of their model show that a significant reduction in $\mathrm{CO} 2$ emissions can be obtained but the price to be paid for this reduction is that the total travel distances as well as the number of routes for the used vehicles increase significantly. A first application of B2C urban distribution problem thought for the design of the logistics systems for medium-large cities in a smart city framework is presented in Carrabs et al. (2014), where the authors face the problem of designing B2C distribution plans keeping large trucks out of the city center. Moreover, thanks to the new real-time systems of air and maritime pollution detection (Negru et al. 2015), it is now possible to identify the most polluted areas of the city and, as a consequence, to adapt both the urban traffic (Gosman et al. 2016) and the transportation of the goods. Usually these tools generate a huge quantity of information (Big Data) that can be processed only with appropriate techniques as described by Pop et al. 2016.

In the present paper, we will make evidence that when the planning of urban distribution tours is made including environmental aspects in the cost function to be minimized,

Table 1 Example of an Italian urban street constraint and time window limitation for the commercial vehicles

\begin{tabular}{llll}
\hline Class of weight $(w)$ & Street availability & Emission type $(e)$ & Time limit circulation \\
\hline$w_{1}$ : trucks $\leq 3,5$ tons & No limit & $e$ : Euro 2 and Euro 3 & From 8 p.m. to 7 a.m. From 10 a.m. to 4 p.m. \\
& & $e 2$ : Euro 4, Euro 5, Euro 6 & From 0 a.m. to 6 p.m. From 7 to 12 p.m. \\
$w_{2}:$ trucks $\leq 5,6$ tons & No alley & $e 3$ : Diesel engine & From 7 p.m. to 7 a.m. \\
& & $e 4:$ Electric engine, GPL, hybrid, methane & No limit \\
$w_{3}:$ trucks $\geq 5,6$ tons & Only avenue & $e 4:$ Electric engine, GPL, hybrid, methane & From 7 p.m. to 7 a.m.
\end{tabular}


the choice of the selected vehicles changes accordingly. In particular, we will show that the optimal solution obtained considering at the same time the travel and pollution costs is better than that obtained by adding the pollution cost to the optimal solution obtained considering only the traveling cost.

The reminder of the paper is organized as follows. A detailed description of the proposed variant of the VRP is given in Sect. 2, together with the description of the daily shift organization and the literature about the problem. Section 3 reports the proposed mixed integer linear programming (MILP) formulation together with the required notation. Section 4 reports on some computational experiments, based on both random instances and real cases, and comments on the results. Finally, outlines for future works are presented in Sect. 5.

\section{The main characteristics of the proposed VRP problem and the underlying network model}

As it has been previously mentioned, the present e-delivery problem in urban area can be seen as an extension of the classical VRP, encountered very frequently in making decisions about the distribution of goods and services.

Let $G=(N, A)$ be a direct graph, where $N$ is the set of $n$ nodes and $A$ is the set of $m$ arcs. The set of nodes consists of a central depot $d$ and a set of customers $U$, where goods have to be delivered. A demand of $g_{i}$ units of goods is associated with each customer $i \in U$.

Given a set $V$ of vehicles, homogeneous or not, with known capacities, the Street Constrained Vehicle Routing Problem (SCVRP) consists of finding a set of routes in $G$, originating and terminating at the central depot $d$ and servicing all the customers exactly once, by a single vehicle, in such a way to satisfy the customer demand. Moreover, since we face the problem in an urban area, we have to take in account even the constraints on the streets, which specify, for each street, which kind of vehicles can cross it. Note that these last constraints arise in practice for different reasons, as the government traffic restrictions of the urban area or the size of the vehicles. For instance, there are streets with flyover forbidden to some vehicles, due to their height.

\subsection{The daily shift organization}

Dealing with B2C urban grocery, we assume that the delivering window is specified in advance, so that customers confirm their orders through the e-channel only if they agree with the proposed delivering time. Note that the available time window for a client is defined with respect to the allowed urban time limits for the commercial vehicles (see Table 1) and the daily working schedules of the e-grocery shipping com- pany under consideration. In particular, taking into account the daily shipping timetable of the main Italian grocery companies (usually split into five shifts from 11 a.m. to 9 p.m.), without loss of generality we assume that the delivery window of each client coincides with the second next available daily shift from the emission order time. As an example, orders put in the e-channel from 1 to 3 p.m. will be delivered between 5 and 7 p.m., while orders sent from 7 to 12 p.m. will be delivered the next day between 11 a.m. and 1 p.m. together with all orders coming from 0 to 7 a.m.

Let us hence have the pair $\left(g_{i}, t_{i}\right)$ of values associated with each customer $i \in U$ representing, respectively, the required amount of goods and the emission order time. Since, as it has been already said, customers agree with the proposed delivering schedule, let $s_{i}$ be the latest delivering time of goods $g_{i}$ ordered at time $t_{i}, \forall i \in U$. Table 2 reports an example of the five daily shifts considered in the present fleet plans delivery decision problem. Note that columns $w_{v}$ and $e_{v}$ report the set of vehicles available in the corresponding daily time period with respect to their weight and emission pollution class, as reported in Table 1.

\subsection{The fleet vehicles characteristics}

In the present paper, we have an additional constraint associated with the daily circulation of the vehicles in urban areas; in particular, due to the need of limiting the fuel emission, to deliver goods by commercial vehicles is allowed only in some daily periods. Further, we have the so-called street constraints, for which the vehicle circulation in some zones or roads of a city is limited to a restricted class of weight of the vehicles. In order to be able to reflect in the classical VRP models the above limitations for the fleet of vehicles, a quadruple of parameters $(w, e, q, c)$ is associated with each vehicle $v \in V$ involved in the distribution process; such parameters specify, respectively, the class of weight, pollution emission type, capacity and cost $/ \mathrm{km}$ of the vehicle. We denote $F$ the set of groups of homogeneous vehicles according to parameters $(w, e, q, c)$. In particular, following the class of weight and the emission type of the vehicles reported in Table 1, we assume that the fleet of vehicles is split into five groups of homogeneous vehicles, that is, $F=$ $\left\{f_{1}, f_{2}, \ldots, f_{5}\right\}$. For example, $f_{1}$ and $f_{2}$ consist of trucks having less than 3.5 tons and emission type 2 and e 1 , respectively, while $f_{5}$ consists of trucks heavier than 5.6 tons with electric engine, GPL, hybrid or methane (emission type e4). Further, we assume that if $i<j$ the vehicles belonging to group $f_{i}$ are less polluting than the vehicles of group $f_{j}$, while the vehicles of the same group have the same characteristics and then are considered equivalent. Note that here the class of weight of the vehicles determines the subset of urban streets that are inhibited to them; instead, the type of the vehicles refers to their category in terms of polluting 
Table 2 Five time daily shifts defining the planning horizon of our decision problem

\begin{tabular}{lllll}
\hline Shift & $w_{v}$ & $e_{v}$ & $s_{i}$ & $t_{i}$ \\
\hline 11 a.m.-1 p.m. & $w 1$ & $e 1, e 2$ & 1 p.m. & 5-12 p.m. the day before 0-7 a.m. \\
& $w 2$ & $e 4$ & & \\
1-3 p.m. & $w 1$ & $e 1, e 2$ & 3 p.m. & 7-11 a.m. \\
& $w 2$ & $e 4$ & & \\
3-5 p.m. & $w 1$ & $e 2$ & 5 p.m. & 11 a.m.-1 p.m. \\
& $w 2$ & $e 4$ & & \\
5-7 p m. & $w 1$ & $e 2$ & 7 p.m. & \\
& $w 2$ & $e 4$ & & $3-5$ p.m. \\
$7-9$ p.m. & $w 1$ & $e 1, e 2$ & 9 p.m. & \\
& $w 2$ & $e 3, e 4$ & & \\
& $w 3$ & $e 4$ & & \\
\hline
\end{tabular}

emissions, for which only in some daily time periods their circulation is allowed. Moreover, it is worth noting that the capacity $q$ of a vehicle, a part from the maximum loading, implies also the possibility to load special goods, such as fresh, refrigerated, or perishable goods. Finally, note that the cost of each vehicle depends on both its class of weight $(w)$ and the pollution emission according to its type $(e)$.

For this purpose, a pair $(l, w)$ of labels is associated with each $\operatorname{arc}(i, j) \in A$ representing, respectively, the length of the corresponding roads and its type, according to its width, following the corresponding parameter $w$ associated with the type of the vehicles. Without loss of generality, we assume that the labels are ordered in a decreasing order according to the width of the street. For example, if there are three types of roads (avenue, street, alley), there are three different labels, namely $w_{1}, w_{2}$ and $w_{3}$. The vehicles having label $w_{1}$ can travel everywhere, vehicles with label $w_{2}$ can travel along roads labeled $w_{2}$ and $w_{3}$, while vehicles with label $w_{3}$ are allowed to pass only through roads of type $w_{3}$. An example of urban network model representing the present problem is reported in Fig. 1.

\subsection{Related work}

In this section, we report the literature regarding the variants of the VRP that are similar to the SCVRP. As it has been previously described, the SCVRP consists of defining a set of routes starting and ending into the depot to deliver the required goods to the customers minimizing the total travel cost. Each route has to respect the capacity constraint of the vehicle assigned to it and, additionally, must meet the size constraint on the streets, which specify which kind of vehicle can cross them. The customer demands and the parameters associated with the vehicles are assumed to be known within each daily shift. The considered monetary cost components, to be minimized, take into account traveling, loading and environmental issues. Note that this last component is con-

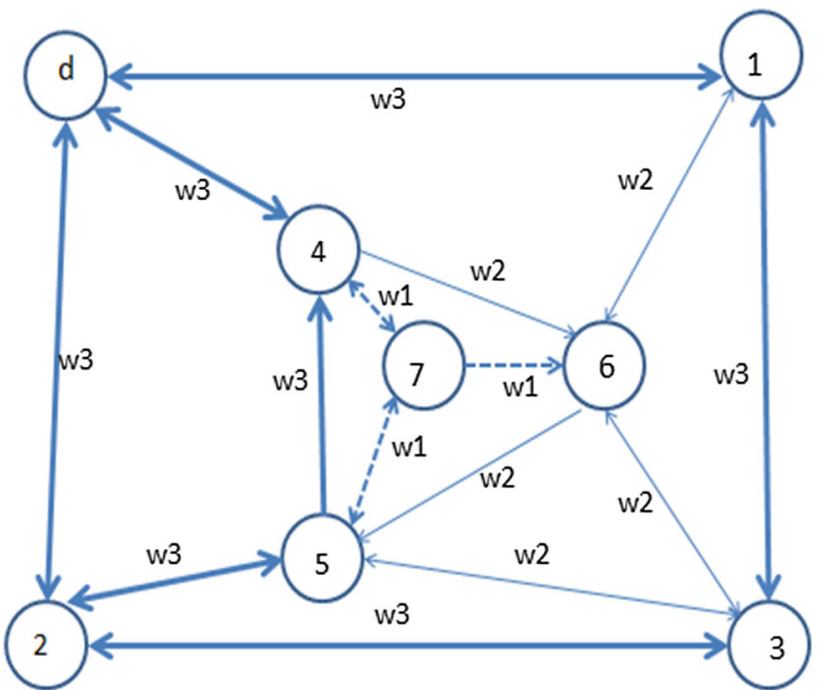

Fig. 1 Example of the underlying network model

sidered very recently in the literature (see e.g., Sarkis et al. 2011, for a literature review). An overview of eco-sustainable supply chain management aspects is reported in Govindam and Sarkis (2014).

Vehicle routing is a classical field of combinatorial optimization, and many are the valid modeling and algorithmic contributions to this NP-complete problem (Lenstra and Rinnooy 1981). For surveys on the state of the art in VRP research, we recommend the ones by Cordeau et al. (2002) and Toth and Vigo (2015), which describe both exact and heuristic methods, and the book by Braysy and Gendreau (2005), which focuses on meta-heuristics. The SCVRP is a variant of the classical VRP, and to the best of our knowledge there is no literature on this problem. The most similar problem to the SCVRP is the Capacitated Arc Routing Problem with Vehicle Site Dependencies (CARP-VSD) introduced by Sniezek et al. (2001), where the authors proposed a mathematical model that was successfully applied in Philadelphia; 
in this problem, street constraints for the vehicles are given and no fixed costs are considered.

There are other variants of the VRP that have applications in $\mathrm{B} 2 \mathrm{C}$. For instance, to the $\mathrm{B} 2 \mathrm{C}$ problem is relevant the "dynamic vehicle routing problem", in which new orders arrive when the routes are already defined. The dynamic nature of e-fulfillment is emphasized in Du et al. (2005), where a combination of several existing algorithms for quick response delivery in an online $\mathrm{B} 2 \mathrm{C}$ environment is proposed. In Francis and Smilowitz (2006), the "periodic VRP" with service choice is studied; in this problem, delivery routes must be constructed for multiple periods and the delivery frequency is a decision variable.

In other variants of the VRP, constraints are associated with the zone of the city available to the vehicles, according to their characteristics. Regarding variants of the VRP with constraints between vehicles and customers, one of the more studied problems is the site-dependent VRP (SDVRP). In this problem, a fleet of heterogeneous vehicles have to satisfy the demand of the customers, but there is a compatibility relationship between the customers and the vehicles. For instance, some customers located in congested urban areas can be served only by small-capacity vehicles, while some customers located in suburban areas can be served by any type of vehicle. Nag et al. (1988) were the first to face the SDVRP. Successively, a heuristic was proposed by Chao et al. (1999) and a tabu search by Cordeau and Laporte (2001) and by Chao and Liou (2005). Finally, a heuristic algorithm composed by three phases (clustering, assigning of vehicle and finding the best routes by combining feasible solution) was recently introduced by Yusuf (2014). Variants of VRP with real-life constraints are considered in Confessore et al. (2008); for these variants, an evolutionary algorithm and a heuristic approach are proposed. An interesting variant of SCVRP requires that the truck starts the tour from the depot but without goods. These goods will be loaded in several locations of the city, and when the loading operations are completed, the unloading operations to the customers are carried out. In this case, each route can be optimized by solving the double traveling salesman problem (Petersen and Madsen 2009; Carrabs et al. 2013).

\section{The MILP model}

Since the demand of each customer is satisfied in a daily shift fixed a priori, let us split the set $U$ of customers into five subsets $U_{t}, t=1, \ldots, 5$, one for each daily shift in which the demands of customers assigned to them must be satisfied. In this way, the computation of a solution for each daily shift is independent of the other daily shifts; therefore, we can solve the B2C problem individually for each daily shift. In particular, we introduce a MILP model that, fixed a daily shift $t$, computes the optimal solution for the customers $U_{t} \subseteq U$ assigned to shift $t$. It is worth reminding that all customers $i \in U_{t}$ have already accepted their latest daily delivering time $s_{i}$, which is implicitly satisfied in the current shift $t, t=$ $1, \ldots, 5$.

The required notations and decision variables of the proposed MILP model for the variant of the SCVRP tailored for the $\mathrm{B} 2 \mathrm{C}$ e-commerce grocery distribution problem under consideration are the following:

Sets and indices:

$G$ digraph representing the urban distribution network

A set of arcs

$N \quad$ set of nodes

$U_{t} \subset N$ subset of customers assigned to the daily shift $t$

$d \quad$ depot node

$F \quad$ set of homogeneous groups of the fleet of vehicles

\section{Constants:}

$n \quad$ number of nodes (including the depot)

$m \quad$ number of arcs

$g_{i} \quad$ goods demand of customer $i \in U$

$Q_{v} \quad$ capacity of vehicles belonging to group $f_{v}$

$m_{v} \quad$ number of available vehicles belong to group $f_{v}$

$\phi_{v} \quad$ fixed cost of a vehicle of group $f_{v}$

$c_{i j v} \quad$ cost to pay for a vehicle of group $f_{v}$ to travel along $\operatorname{arc}(i, j) \in A$

$s_{i j v} \quad$ it is equal to 1 if a vehicle of group $f_{v}$ can travel along $\operatorname{arc}(i, j) \in \mathrm{A}$ and 0 otherwise.

Note that value $c_{i j v}$ is computed according to the group $f_{v}$ and the emission type $e_{v}$ of vehicle $v$ and the length $l_{i j}$ of arc $(i, j) \in A$; consequently, in the following MILP model the unit cost coefficient $c_{i j v}$ of the objective function takes into a proper account all the desired distribution cost components, including environmental issues.

The following variables are then defined:

$x_{i j v}$ binary variable that assumes value equal to 1 if a vehicle of group $f_{v}$ travels from $i$ to $j$ and 0 otherwise;

$y_{i j v}$ continuous variable representing the flow of goods carried by a vehicle of group $f_{v}$ from node $i$ to node $j$.

Having in mind the above notation, let us now give the proposed MILP formulation for the present problem related to the fleet distribution planning for a given daily shift $t$.

$\min \sum_{v \in F} \sum_{j \in N} \phi_{v} x_{d j v}+\sum_{v \in F} \sum_{(i, j) \in A} c_{i j v}, x_{i j v}$ 


$$
\begin{aligned}
& \sum_{v \in F} \sum_{j \in N} x_{i j v}=1 \quad \forall j \in U_{t} \\
& \sum_{v \in N} x_{i k v}-\sum_{j \in N} x_{k j v}=0 \quad \forall k \in N \backslash\{d\}, \forall v \in F \\
& x_{i j v} \leq s_{i j v} \quad \forall i, j \in N, \forall v \in F \\
& \sum_{j \in N \backslash\{d\}} x_{d j v} \leq m_{v} \quad \forall v \in F \\
& \sum_{v \in F} \sum_{j \in N \backslash\{d\}} y_{d j v} \geq \sum_{i \in N \backslash\{d\}} g_{i} \\
& \sum_{i \in N} y_{i k v}-\sum_{j \in N} y_{k j v} \\
& =g_{k} \sum_{i \in N} x_{i k v} \quad \forall k \in N \backslash\{d\}, \forall v \in F \\
& y_{i j v} \leq\left(Q_{v}-g_{i}\right) x_{i j v} \quad \forall(i, j) \in A, \forall v \in F \\
& y_{i j v} \geq g_{j} x_{i j v} \quad \forall(i, j) \in A, \forall v \in F \\
& x_{i j v} \in\{0,1\} \quad \forall i, j \in N, \forall v \in F \\
& y_{i j v} \geq 0 \quad \forall(i, j) \in A, \forall v \in F
\end{aligned}
$$

In the above formulation, the objective function (1) minimizes the total distribution cost to serve all customers. Remember that the unit cost coefficient of the vehicles depends on both their class of weight and pollution emission type. Moreover, we consider a fixed cost $\phi_{v}$ associated with the group $f_{v}$ of vehicle $v$.

Constraints (2) and (3) impose that a customer is visited exactly once and that if a vehicle visits a customer, it must also depart from it. Constraints (4) guarantee that each vehicle is allowed to travel only along the streets corresponding to its class of weight. Note that the value of $s_{i j v} \forall i, j \in N$ and $\forall v \in F$ is set in a preprocessing phase according to the urban traffic limitations and government law under consideration. The maximum number of vehicles available for each class of weight is imposed by constraints (5). Constraints (6) force the quantity of goods outgoing from the depot to be at least equal to the sum of the customer demands. Note that it is not required that the vehicles come back to the depot empty. Constraints (7) are the so-called flow-commodity constraints, specifying the difference between the quantity of goods a vehicle carries before and after visiting a node; such difference is equal to the demand of the corresponding customers. Constraints (8) ensure that the vehicle capacity is never exceeded, while constraints (9) guarantee that the value $y_{i j v}$ must be at least equal to $g_{j}$ if a vehicle $v$ crosses arc $(i, j) \in$ A. Finally, (10) and (11) define the decision variables introduced above.

It is important to mention that a relevant problem to face in the formulation of model (1)-(11) concerns the symmetry. In fact, the present formulation relies on a directed graph, while most of the benchmark instances in literature concerning VRP are based on undirected ones. As a consequence, for each feasible solution of the present $\mathrm{B} 2 \mathrm{C}$ problem, there are several equivalent solutions derived just inverting the visiting order of the nodes in the corresponding route. Further, since the convergence of the above MILP model is significantly slowed down by these symmetry problems, we had to face them by adding the so-called symmetry breaking constraints to model (1)-(11). To this end, we impose that, for each route $q$, the identification number of the first visited node, say $i$, should be lower than that of the last visited one, say $j$, that is, we force $i<j, \forall i, j \in U_{t}$ and $\forall i, j \in q$. In this way, we associate with each node $j \in N$ a new integer variable $r_{j} \in[1, n]$, representing the identification number of the first node visited in the route where $j$ is located. The following symmetry breaking constraints are then added to the original formulation:

$$
\begin{aligned}
& r_{j} \geq j \sum_{v \in F} x_{d j v} \quad \forall(d, j) \in A \\
& r_{j} \leq j \sum_{v \in F} x_{d j v}+\left(1-\sum_{v \in F} x_{d j v}\right) n \quad \forall(d, j) \in A \\
& r_{j} \geq r_{i}-\left(1-\sum_{v \in F} x_{i j v}\right) n \quad \forall(i, j) \in A \\
& r_{j} \leq r_{i}+\left(1-\sum_{v \in F} x_{i j v}\right) n \quad \forall(i, j) \in A \\
& r_{i} \leq i \sum_{v \in F} x_{d j v}+\left(1-\sum_{v \in F} x_{i d v}\right) n
\end{aligned}
$$

Constraints (12) and (13) assign value $j$ to variable $r_{j}$ since node $j$ is the first visited node in the route. Constraints (14) and (15) assign value $i$ to variable $r_{j}$ if node $j$ belongs to a route starting with node $i$. Finally, constraints (16) assure that the identification number of the first node of the route is lower than that of the last node on the same route. As it has been already said, note that the above (12)-(16) constraints play the role of making more efficient the computational process of the $\mathrm{B} 2 \mathrm{C}$ problem represented by (1)-(11); in particular, the new added constraints do not impact on the operative scenario of the $\mathrm{B} 2 \mathrm{C}$ problem, that is expressed by constraints (2)-(9).

Further, it is possible to prove that some valid inequalities proposed in literature (Baldacci et al. 2009) for the heterogeneous fleet vehicle routing problem are valid for the B2C too. However, it is out of the scope of this paper to present a branch and cut approach for the B2C and then we do not introduce these valid inequalities in our formulation. 


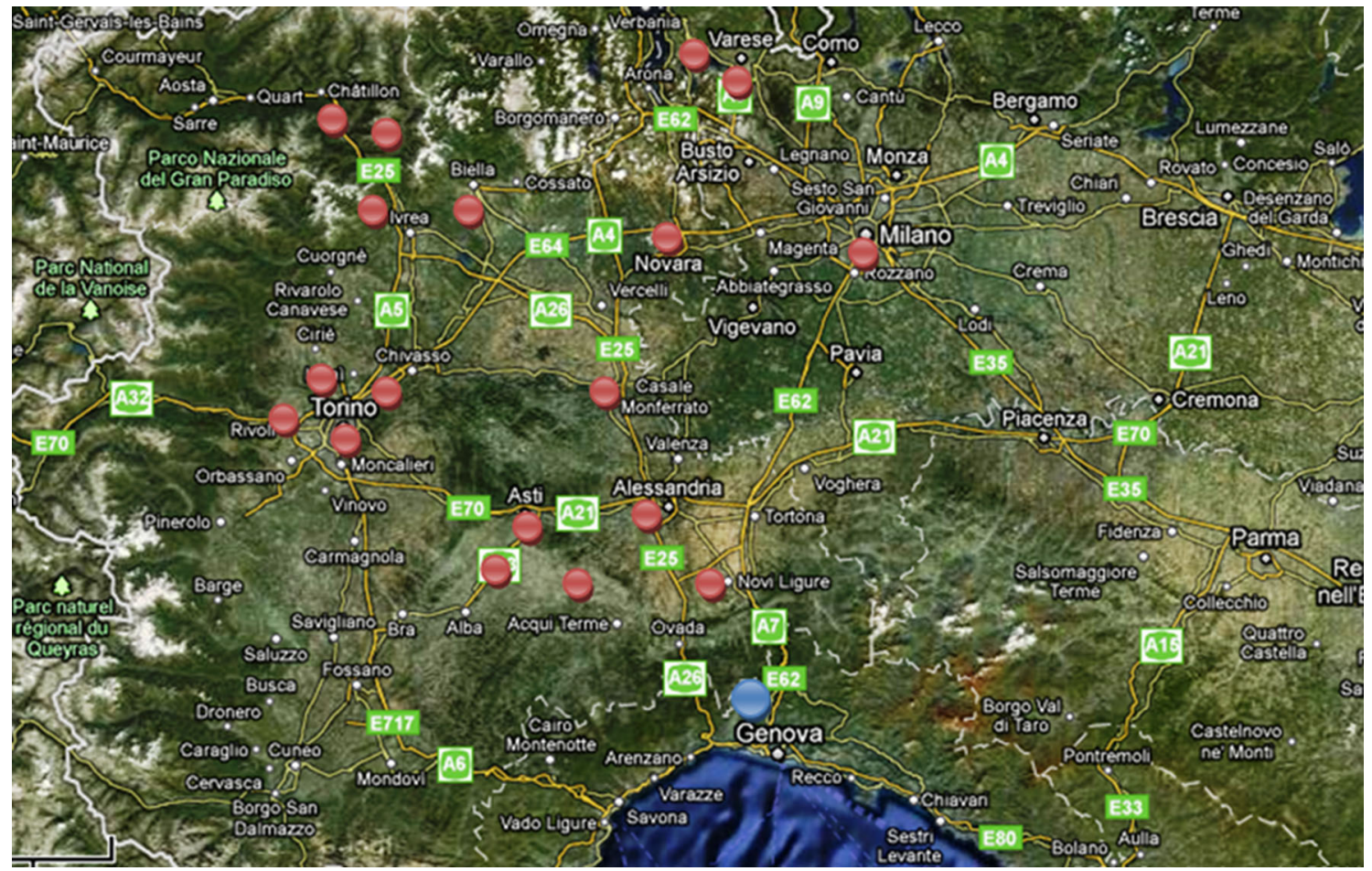

Fig. 2 Map with the location of the depot (blue circle) and of the customers (red circle) (color figure online)

\section{Computational results}

The MILP model proposed in Sect. 3 has been implemented in $\mathrm{C}++$ and solved by CPLEX 12.5 and run on a $2.33 \mathrm{GHz}$ Intel Core2 Q8200 processor (2007). We start our computational test phase by using realistic instances retrieved by the distribution plans of an Italian e-grocery company which depot is located near Genoa. The company has to satisfy the demands of its customers that are located in urban areas of three regions of Italy, namely Lombardy, Piedmont and Aosta valley. Figure 2 shows the positions of the customers.

Table 3 gives the location and demand, expressed in number of rolls, of each customer. The reported data refer to the latest delivery shift (i.e., from 5 to 7 p.m., see Table 2) of a weekly day. A number $(1,2,3)$ is associated with each customer expressing the maximum class of width of the vehicles allowed for visiting it; more precisely, value 1 means that only the tightest vehicle can visit the corresponding customer, while value 3 means that even the largest vehicle can visit it. We identify the vehicles that can cross an arc $(i, j)$ by choosing the minimum width associated with $i$ and $j$.

In this scenario, the available fleet of the company consists of three vehicles with the same capacities ( 21 rolls) but different width, that is, 1, 2, and 3, respectively. In order to compare our solution with that actually running by the company, in the objective function (1) we consider only the traveling distance and costs associated with the size (width) of the vehicles; in
Table 3 Delivering information about customers

\begin{tabular}{llll}
\hline Customer & City & Demand & Width \\
\hline 1 & Turin & 1 & 2 \\
2 & Turin & 1 & 2 \\
3 & Milan & 2 & 2 \\
4 & Varese & 2 & 2 \\
5 & Varese & 1 & 2 \\
6 & Alessandria & 1 & 2 \\
7 & Alessandria & 1 & 1 \\
8 & Biella & 1 & 3 \\
9 & Turin & 1 & 1 \\
10 & Aosta & 1 & 1 \\
11 & Turin & 1 & 3 \\
12 & Asti & 1 & 3 \\
13 & Asti & 1 & 2 \\
14 & Alessandria & 3 & 3 \\
15 & Asti & 2 & 3 \\
16 & Novara & 1 & 3 \\
17 & Aosta & 2 & 2 \\
18 & Turin & 2 & 2 \\
\hline
\end{tabular}

fact, no pollution factors are considered in the practical case. The optimal solution is found by the proposed MILP model in $12 \mathrm{~s}$, obtaining an objective function value corresponding to $1033 \mathrm{~km}$; such distance is the sum of the length of two 
Table 4 Information about the instances used in the computational test bed

\begin{tabular}{|c|c|c|c|c|c|c|c|c|c|c|c|}
\hline \multicolumn{5}{|c|}{3 Groups of vehicles } & \multicolumn{7}{|c|}{5 Groups of vehicles } \\
\hline \multirow[b]{2}{*}{ Id } & \multirow[b]{2}{*}{ Name } & \multicolumn{2}{|l|}{$w_{1}$} & \multirow{2}{*}{$\begin{array}{l}w_{2} \\
f_{3}\end{array}$} & \multirow[b]{2}{*}{ Id } & \multirow[b]{2}{*}{ Name } & \multicolumn{2}{|l|}{$w_{1}$} & \multicolumn{2}{|l|}{$\underline{w_{2}}$} & \multirow{2}{*}{$\begin{array}{l}w_{3} \\
f_{5}\end{array}$} \\
\hline & & $f_{1}$ & $f_{2}$ & & & & $f_{1}$ & $f_{2}$ & $f_{3}$ & $f_{4}$ & \\
\hline 1 & SCG_10_45_v3_s1 & 100 & 100 & 7 & 25 & SCG_10_45_v5_s181 & 100 & 100 & 34 & 34 & 7 \\
\hline 2 & SCG_10_45_v3_s7 & 100 & 100 & 14 & 26 & SCG_10_45_v5_s187 & 100 & 100 & 34 & 34 & 14 \\
\hline 3 & SCG_10_45_v3_s13 & 100 & 100 & 7 & 27 & SCG_10_45_v5_s193 & 100 & 100 & 34 & 34 & 3 \\
\hline 4 & SCG_20_190_v3_s19 & 100 & 100 & 48 & 28 & SCG_20_190_v5_s199 & 100 & 100 & 35 & 35 & 8 \\
\hline 5 & SCG_20_190_v3_s25 & 100 & 100 & 12 & 29 & SCG_20_190_v5_s205 & 100 & 100 & 24 & 24 & 4 \\
\hline 6 & SCG_20_190_v3_s31 & 100 & 100 & 15 & 30 & SCG_20_190_v5_s211 & 100 & 100 & 35 & 35 & 4 \\
\hline 7 & SCG_30_435_v3_s37 & 100 & 100 & 28 & 31 & SCG_30_435_v5_s217 & 100 & 100 & 36 & 36 & 1 \\
\hline 8 & SCG_30_435_v3_s43 & 100 & 100 & 3 & 32 & SCG_30_435_v5_s223 & 100 & 100 & 25 & 25 & 3 \\
\hline 9 & SCG_30_435_v3_s49 & 100 & 100 & 9 & 33 & SCG_30_435_v5_s229 & 100 & 100 & 21 & 21 & 4 \\
\hline 10 & SCG_40_780_v3_s55 & 100 & 100 & 25 & 34 & SCG_40_780_v5_s235 & 100 & 100 & 72 & 72 & 9 \\
\hline 11 & SCG_40_780_v3_s61 & 100 & 100 & 22 & 35 & SCG_40_780_v5_s241 & 100 & 100 & 33 & 33 & 3 \\
\hline 12 & SCG_40_780_v3_s67 & 100 & 100 & 16 & 36 & SCG_40_780_v5_s247 & 100 & 100 & 27 & 27 & 9 \\
\hline 13 & SCG_50_1225_v3_s73 & 100 & 100 & 14 & 37 & SCG_50_1225_v5_s253 & 100 & 100 & 38 & 38 & 14 \\
\hline 14 & SCG_50_1225_v3_s79 & 100 & 100 & 4 & 38 & SCG_50_1225_v5_s259 & 100 & 100 & 58 & 58 & 1 \\
\hline 15 & SCG_50_1225_v3_s85 & 100 & 100 & 2 & 39 & SCG_50_1225_v5_s265 & 100 & 100 & 23 & 23 & 4 \\
\hline 16 & SCG_60_1770_v3_s91 & 100 & 100 & 12 & 40 & SCG_60_1770_v5_s271 & 100 & 100 & 47 & 47 & 1 \\
\hline 17 & SCG_60_1770_v3_s97 & 100 & 100 & 2 & 41 & SCG_60_1770_v5_s277 & 100 & 100 & 32 & 32 & 8 \\
\hline 18 & SCG_60_1770_v3_s103 & 100 & 100 & 20 & 42 & SCG_60_1770_v5_s283 & 100 & 100 & 14 & 14 & 6 \\
\hline 19 & SCG_70_2415_v3_s109 & 100 & 100 & 9 & 43 & SCG_70_2415_v5_s289 & 100 & 100 & 28 & 28 & 2 \\
\hline 20 & SCG_70_2415_v3_s115 & 100 & 100 & 3 & 44 & SCG_70_2415_v5_s295 & 100 & 100 & 34 & 34 & 16 \\
\hline 21 & SCG_70_2415_v3_s121 & 100 & 100 & 4 & 45 & SCG_70_2415_v5_s301 & 100 & 100 & 60 & 60 & 11 \\
\hline 22 & SCG_80_3160_v3_s127 & 100 & 100 & 23 & 46 & SCG_80_3160_v5_s307 & 100 & 100 & 62 & 62 & 12 \\
\hline 23 & SCG_80_3160_v3_s133 & 100 & 100 & 12 & 47 & SCG_80_3160_v5_s313 & 100 & 100 & 33 & 33 & 7 \\
\hline 24 & SCG_80_3160_v3_s139 & 100 & 100 & 26 & 48 & SCG_80_3160_v5_s319 & 100 & 100 & 28 & 28 & 4 \\
\hline
\end{tabular}

independent tours performed by two vehicles of width 1 and 2 , respectively. This solution is significantly better than the solution currently used by company, which has a total length of $1325.9 \mathrm{~km}$.

We solved the same instance by considering the customers demands of a peak shift on Saturday, corresponding to 52 rolls. Despite the customer demands are higher, the model found the optimal solution, corresponding to $1426 \mathrm{~km}$, in $117 \mathrm{~s}$. Note that the solution is worse than that obtained in the previous case $(1033 \mathrm{~km})$; in fact, in this last case the model is forced to use all three available vehicles for being able to satisfy the higher customer demands, changing, consequently, the routes of the vehicles from the ones of the original instances.

Since there are no benchmark instances in the literature for the present B2C problem, we had the need of generating a new set of instances. Then, we carried out the computational tests on graphs having from 10 to 80 nodes (value of $n$ ) representing the depot and the customers. For each size $n$, three instances are generated, with a different assignment of the fixed and traveling costs, established according to the size and type of set $F$ representing the groups of homogeneous vehicles.

All the instances used for the computational tests are listed in Table 4. The instances refer to a daily shift described in Table 2 and differ in the number of groups of available vehicles, which are either 3 or 5. In more details, the first 24 instances (with id from 1 to 24 ) refer to the daily shift from 1 to 3 p.m., when only vehicles of groups $f_{1}, f_{2}$ (class of weight $w_{1}$ ) and $f_{3}$ (class of weight $w_{2}$ ) can circulate. Instead, the last 24 instances (with id from 25 to 48 ) refer to the daily shift from 7 to 9 p.m., when the circulation is allowed to all groups of vehicles (groups $f_{1}, f_{2}$ for the class of weight $w_{1}$, groups $f_{3}, f_{4}$ for the class of weight $w_{2}$ and group $f_{5}$ for the class of weight $w_{3}$ ). Table 4 reports the name of each instance, in the format SCG_\# nodes_\# edges_\# vehicles_seed, and the number of arcs, in percentage, on which each group of vehicles can travel. More precisely, for the groups $f_{1}, f_{2}$ of the first instance, the value 100 means that the vehicles can cross any edge of the network, while only the $7 \%$ of the edge in $G$ can be crossed by the vehicles of group $f_{3}$ due to their class of weight $w_{2}$. 
Table 5 Test results on the instances with 3 groups of homogeneous vehicles

\begin{tabular}{llllllr}
\hline Id & $n$ & $m_{1}, m_{2}, m_{3}$ & Obj & $k_{1}, k_{2}, k_{3}$ & Time & GAP $(\%)$ \\
\hline 1 & 10 & $3,5,2$ & 310 & $3,1,1$ & 0.07 & 0.00 \\
2 & 10 & $2,3,5$ & 343 & $2,1,2$ & 0.11 & 0.00 \\
3 & 10 & $3,5,2$ & 288 & $3,1,1$ & 0.04 & 0.00 \\
4 & 20 & $2,2,4$ & 803 & $2,0,3$ & 113.21 & 0.00 \\
5 & 20 & $3,2,3$ & 883 & $3,1,2$ & 37.62 & 0.00 \\
6 & 20 & $2,5,3$ & 978 & $2,2,2$ & 1084.79 & 0.00 \\
7 & 30 & $2,5,10$ & 1979 & $2,2,4$ & $10,810.05$ & 1.35 \\
8 & 30 & $8,5,2$ & 1422 & $7,0,1$ & 603.93 & 0.00 \\
9 & 30 & $4,3,4$ & 1311 & $4,1,2$ & 467.49 & 0.00 \\
10 & 40 & $5,7,5$ & 3000 & $5,2,3$ & $10,810.10$ & 5.71 \\
11 & 40 & $5,4,7$ & 3184 & $5,2,5$ & $10,810.09$ & 5.29 \\
12 & 40 & $6,4,7$ & 3277 & $6,2,3$ & $10,810.09$ & 7.20 \\
13 & 50 & $4,10,7$ & 4391 & $4,4,4$ & $10,810.13$ & 6.13 \\
14 & 50 & $4,10,5$ & 4783 & $4,5,3$ & $10,810.17$ & 6.05 \\
15 & 50 & $5,14,3$ & 4508 & $5,6,2$ & $10,810.14$ & 7.81 \\
16 & 60 & $4,9,7$ & 6311 & $4,4,4$ & $10,810.22$ & 15.40 \\
17 & 60 & $12,12,4$ & 5364 & $12,2,3$ & $10,810.22$ & 3.80 \\
18 & 60 & $9,5,18$ & 8197 & $9,3,8$ & $10,810.19$ & 8.47 \\
19 & 70 & $5,25,13$ & 10618 & $5,9,6$ & $10,810.28$ & 10.60 \\
20 & 70 & $16,7,7$ & 9421 & $16,0,5$ & $10,810.29$ & 3.18 \\
21 & 70 & $9,8,5$ & 7153 & $9,4,2$ & $10,810.28$ & 16.10 \\
22 & 80 & $4,13,12$ & 16,074 & $4,9,8$ & $10,810.39$ & 15.20 \\
23 & 80 & $8,23,14$ & 14,651 & $8,9,6$ & $10,810.39$ & 19.80 \\
24 & 80 & $7,7,15$ & 11,498 & $7,5,8$ & $10,810.39$ & 14.30 \\
\hline & & & & & &
\end{tabular}

Table 5 reports the results obtained by CPLEX 12.5 using the proposed MILP model on the instances with three groups of vehicles (id 1-24). The first three columns of Table 5 list the instance $(i d)$, the number of nodes $(n)$ and the number of different vehicles available for each group in $F$, respectively. The next three columns, namely $O b j, k_{1}, k_{2}, k_{3}$ and Time, report, respectively, the objective function value, the number of vehicles used, for each considered group, and the required CPU time (in seconds). Note that a threshold of $3 \mathrm{~h}$ was imposed for the resolution of each instance. The last column (GAP) reports the gap between the lower bound computed by CPLEX and the feasible solution computed by our model. Note that a value different from zero means that the proposed MILP model did not find the optimal solution within the fixed threshold limit.

The results of Table 5 show that CPLEX 12.5 is able to solve all instances of the proposed model up to 20 nodes and two instances out of three with 30 nodes within the given time limit. The computational time is negligible for the instances with 10 nodes and is lower than 20 min for all other instances with 20 nodes. Two instances (id 8 and 9) with 30 nodes are solved in less than $10 \mathrm{~min}$, while for the third one (id 7) the time limit is reached with a gap equal to $1.35 \%$. Finally, on the instances from 40 to 80 nodes, the MILP model always reaches the time limit with a gap ranging from 3.18 to $19.80 \%$. Notice that, as we expected, the vehicles of group $f_{2}$ are used only if all the cheapest available vehicles of group $f_{1}$ are chosen. Moreover, at least one vehicle of group $f_{3}$ is always used because, by construction, in set $U$ there are customers with a demand greater than $Q_{2}$. It is interesting to observe that in the instances with at least 60 nodes lower is value $m_{1}$ higher is the value of the gap. Indeed, in instance 16, the gap is $15.40 \%$ and the value of $m 1$ is 4 , while in instances 17 and 18, having the same number of nodes and edges, the gap is 3.80 and $8.47 \%$ and $m_{1}$ is equal to 12 and 9, respectively. Further, in instance 20, having a value of $m_{1}$ equal to 16 , the gap is $3.18 \%$, while in the other two instances having 70 nodes and a lower value of $m_{1}$, the gap is much higher. Our hypothesis is that with high values of $m_{1}$ model (1)-(16) is able to satisfy most of the customers with the cheapest vehicles, thus obtaining good solutions. Indeed, almost always all $m_{1}$ vehicles are used into the solution. Moreover, since these vehicles can cross any edge of the graph, it is easy to build routes for them. Instead, when the value of $m_{1}$ is low, the model is forced to use more expensive vehicles that can cross only some type of edges. This makes more expensive to determine feasible routes for these vehicles slowing down the performance of the model.

Table 6 shows the results of the model when five different groups of vehicles are available. Columns headings are the same as those in Table 5. Also in this case, all instances up to 20 nodes are optimally solved by the proposed model. However, these instances result harder to solve than those with three types of vehicles. Indeed, in the worst case, instances with 20 nodes require $70 \mathrm{~min}$ (instance id 29) while the same instances of Table 5 require less than $20 \mathrm{~min}$. Moreover, there are not instances with 30 nodes solved within the time limit even if the gap is very low for instances 32 and 33 ( 0.59 and $0.55 \%$, respectively) and equal to $4.35 \%$ for instance 31 .

The gaps on instances with at least 40 nodes range from $3.19 \%$ (instance 35 ) to $26.30 \%$ (instance 46 ). Even in this table, the complexity of the instances seems to depend on the number of cheapest available vehicles $m_{1}$ and $m_{2}$. Indeed, in the case of instance 46 , the gap is $26.30 \%$ because the value of $m_{1}$ and $m_{2}$ is very low, fixed to 3 and 2, respectively. In instance 47 , this gap decreases to $11.50 \%$ because $m_{1}$ grows from 3 to 5 and $m_{2}$ grows from 2 to 15 . Finally, in instance 48 , the gap decreases to $6.84 \%$ because, with $m_{1}=15$ and $m_{2}=4$, there are cheaper vehicles available to build the solution. Actually, there are so much vehicles of group $f_{1}$ that the vehicles of $f_{2}$ are not used.

These results prove that the number of vehicle groups impacts on the effectiveness of the proposed MILP model; in fact, it results harder to find the optimal solution when 
Table 6 Test results on the instances with 5 vehicles

\begin{tabular}{|c|c|c|c|c|c|c|}
\hline Id & $\mathrm{n}$ & $m_{1}, m_{2}, m_{3}, m_{4}, m_{5}$ & Obj & $k_{1}, k_{2}, k_{3}, k_{4}, k_{5}$ & Time & GAP $(\%)$ \\
\hline 25 & 10 & $2,5,3,2,3$ & 872 & $2,2,3,0,2$ & 0.02 & 0.00 \\
\hline 26 & 10 & $2,6,2,2,6$ & 1123 & $2,2,2,0,3$ & 0.02 & 0.00 \\
\hline 27 & 10 & $3,3,3,4,2$ & 736 & $3,1,3,1,1$ & 0.02 & 0.00 \\
\hline 28 & 20 & $3,2,2,2,4$ & 1667 & $3,1,2,0,2$ & 177.77 & 0.00 \\
\hline 29 & 20 & $3,3,2,2,2$ & 1449 & $3,2,2,0,1$ & 4182.04 & 0.00 \\
\hline 30 & 20 & $3,3,3,2,2$ & 1471 & $3,2,3,0,1$ & 289.43 & 0.00 \\
\hline 31 & 30 & $2,3,3,4,2$ & 3384 & $2,2,3,1,1$ & $10,810.10$ & 4.35 \\
\hline 32 & 30 & $5,2,3,5,2$ & 2635 & $5,1,3,1,1$ & $10,810.11$ & 0.59 \\
\hline 33 & 30 & $4,2,2,2,3$ & 2748 & $4,0,2,0,2$ & $10,810.09$ & 0.55 \\
\hline 34 & 40 & $2,4,3,4,4$ & 5058 & $2,3,3,1,2$ & $10,810.14$ & 5.14 \\
\hline 35 & 40 & $2,4,4,2,2$ & 3486 & $2,3,4,0,1$ & $10,810.16$ & 3.19 \\
\hline 36 & 40 & $3,3,2,3,5$ & 4951 & $3,3,2,1,3$ & $10,810.14$ & 3.83 \\
\hline 37 & 50 & $4,5,2,5,10$ & 7731 & $4,3,2,2,4$ & $10,810.25$ & 6.00 \\
\hline 38 & 50 & $2,3,3,12,4$ & 9355 & $2,3,3,7,2$ & $10,810.25$ & 8.95 \\
\hline 39 & 50 & $5,11,4,2,3$ & 7224 & $5,5,4,0,2$ & $10,810.24$ & 3.53 \\
\hline 40 & 60 & $6,4,7,4,4$ & 12,554 & $6,2,7,1,2$ & $10,810.35$ & 12.50 \\
\hline 41 & 60 & $6,7,6,2,4$ & 9248 & $6,3,5,0,3$ & $10,810.34$ & 6.83 \\
\hline 42 & 60 & $8,7,5,2,5$ & 12,434 & $8,4,4,0,3$ & $10,810.37$ & 7.02 \\
\hline 43 & 70 & $6,6,6,6,5$ & 14,173 & $6,5,6,4,2$ & $10,810.54$ & 17.70 \\
\hline 44 & 70 & $3,15,3,2,11$ & 19,515 & $3,11,3,0,7$ & $10,810.52$ & 9.51 \\
\hline 45 & 70 & $4,5,3,14,15$ & 19,836 & $4,5,3,6,6$ & $10,810.49$ & 10.30 \\
\hline 46 & 80 & $3,2,5,9,11$ & 36,401 & $3,2,5,5,8$ & $10,810.68$ & 26.30 \\
\hline 47 & 80 & $5,15,7,10,13$ & 27,356 & $5,8,7,2,6$ & $10,810.73$ & 11.50 \\
\hline 48 & 80 & $15,4,5,6,8$ & 15,427 & $14,0,5,2,4$ & $10,810.74$ & 6.84 \\
\hline
\end{tabular}

the number of vehicle' groups grows. It is interesting to observe that the best results are obtained by the proposed MILP model in the instances where some groups of vehicles are not used. As an example, let us consider the instances with 30 nodes reported in Table 6. The lowest gap occurs in instance 33, where no vehicle of groups $f_{2}$ and $f_{4}$ is used. In the instances with 40 and 50 nodes, the lowest gap occurs in instances 35 and 39, respectively, where no vehicle of group $f_{4}$ is used. The same holds for the remaining instances. This occurs because when it is possible to avoid the use of a group of vehicles, the symmetry in the problem is reduced, thus allowing a faster convergence of the resolution method.

Finally, in order to evaluate the impact of the pollution costs on the solution found and the vehicles chosen, we carried out further computational tests on the largest instances optimally solved by the proposed model using CPLEX 12.5. In particular, we duplicated the instances with 20 nodes and fixed the cost of the arcs equal to the distance between the nodes; in this way, no pollution costs are considered in these instances. We compare the solutions found with and without the pollution cost in Table 7 .

In Table 7, instances 1 and 4 are identical a part from the arc cost, that is, respectively, equal to the pollution and traveling (distance) cost. The same holds for the couples of
Table 7 Test results on the instances with 20 nodes where the cost of edges is pollution costs and distance cost, respectively

\begin{tabular}{llllr}
\hline ID & Edge cost & Obj & Vehicles used & \multicolumn{1}{c}{ Time } \\
\hline 3 Group vehicles & & & \\
1 & Pollution & 803 & $2,0,3$ & 113.21 \\
2 & Pollution & 883 & $3,1,2$ & 37.62 \\
3 & Pollution & 978 & $2,2,2$ & 1084.79 \\
4 & Distance & 596 & $2,0,3$ & 37.04 \\
5 & Distance & $653(923)$ & $3,0,2$ & 135.72 \\
6 & Distance & 688 & $2,2,2$ & 70.14 \\
5 Group vehicles & & & \\
7 & Pollution & 1667 & $3,1,2,0,2$ & 177.77 \\
8 & Pollution & 1449 & $3,2,2,0,1$ & 4182.04 \\
9 & Pollution & 1471 & $3,2,3,0,1$ & 289.43 \\
10 & Distance & $938(1804)$ & $3,0,2,0,2$ & 93.12 \\
11 & Distance & $958(1496)$ & $3,1,2,0,1$ & 1254.46 \\
12 & Distance & $864(1545)$ & $3,1,3,0,1$ & 197.77 \\
\hline
\end{tabular}

instances 2 and 5, 3 and 6 and so on. Obviously, since the pollution costs are always greater than the distance costs, the objective function value of the instances with the pollution 
costs is always greater than that of the instances with the distance cost. However, we are not interested in the comparison of the objective function values; our goal here is to verify whether there are couples of instances where, due to the pollution costs, the fleet of vehicles used changes. Readers can note that this is the case for the couple of instances 2 and 5. In particular, the model solves instance 5 without using vehicles of group 2; this means that sometimes, due to the pollution costs, it is cheaper to satisfy the demand of very few customers by using one more vehicle rather than increasing the length of a route. These results show how the pollution costs have a significant impact on the solutions produced. Indeed, the request of one more vehicle needed every day, in the considered daily shift, has a significant relevance for the costs of the grocery company.

Another aspect that we want to highlight is that the solutions found by including only the traveling cost in the objective function (1) are not optimal if they have to be evaluated also in term of pollution cost. This is a very important aspect since it shows that solving a classical VRP by neglecting the pollution costs does not produce good solutions for the present problem. To give a better evidence of this result, let us refer to instance 5; in column $\mathrm{Obj}$, we report the optimal value (653) obtained considering only the distance cost compared with the value (between bracket) computed including a posteriori the pollution cost; note that this last value (923) is higher than the optimal solution (883) found by the proposed model taking into account the pollution cost. The pollution cost of instances 4 and 6 is not reported because the route found is the same of that obtained for instances 1 and 3 .

Regarding the computational time, usually it is easier to find the optimal solution when only the distance cost is considered.

The results concerning the instances with 5 groups of vehicles are even more interesting. In fact, with more groups of vehicles, there are more choices in the construction of the solutions; therefore, the vehicles used in instances 7,8 , and 9 are different from the vehicles used in instance 10,11, and 12. In particular, as already seen for the instances with 3 vehicle groups, the solutions of the instances with the pollution costs tend to use more vehicles and smaller routes due to the arc cost. Moreover, it is interesting to evaluate the solution costs of these instances in term of pollution costs. In particular, the solution of instance 10 , evaluating a posteriori the pollution cost, is equal to 1804 , while the optimal one is 1667 . Analogously, for instance 11 , the solution value is 1496 instead of 1449 and for instance 12 it is 1545 instead of 1471. Regarding the computational time, it is evident that the instances with the distance cost are easier to solve with respect to the instances with the pollution cost.

These results highlight that it is very important to solve the problem taking in account the pollution cost during the construction of the routes. Indeed, by neglecting these costs, the solution is found in less computational time but this solution can be far from the optimal one in term of pollution costs.

\section{Conclusions and outline for future works}

In the online $\mathrm{B} 2 \mathrm{C}$ grocery environment in metropolitan areas, there is the need of an efficient transportation system to support the order deliveries. The model proposed in this work can be used to improve the service quality in $\mathrm{B} 2 \mathrm{C}$, while taking into a proper account the urban traffic limitations aimed at reducing the pollution emission. In particular, the present variant of the VRP finds optimal fleet schedules for single daily shift for serving customers along different routes in a network in which only restricted classes of vehicles are allowed to travel due to their weight and pollution emission.

The computational results show that the solutions found by our model have a significantly lower pollution cost with respect to the other approaches in which this cost is neglected. As a consequence, our approach can represent a valid tool to reduce the pollution in the cities. Moreover, the capacity of selecting the appropriate vehicles for each time shift has a relevant impact for the costs of the grocery company.

Starting from the very promising preliminary computational results presented in this work, the next research direction will be the application of the MILP model on real data coming from a grocery company within a medium-large urban territory. Finally, for larger real cases, we intend to develop efficient metaheuristics able to quickly find good solutions.

\section{Compliance with ethical standards}

Conflict of interest The F. Carrabs declares that he has no conflict of interest. The R. Cerulli declares that he has no conflict of interest. The A. Sciomachen declares that he has no conflict of interest.

Ethical approval This article does not contain any studies with human participants or animals performed by any of the authors.

\section{References}

Agatz NAH, Fleischmann M, van Numen JAEE (2008) E-fulfillment and multi-channel distribution. A review. Eur J Oper Res 187:339356

Baldacci R, Battarra M, Vigo D (2009) Valid inequalities for mix vehicle routing the fleet size and problem with fixed costs. Networks 54(4):178-189

Blumenfeld DE, Burns LD, Diltz JD, Daganzo CF (1985) Analyzing trade-offs between transportation, inventory and production costs on freight networks. Transp Res B 19:361-380

Boyer KK, Hult GT, Frohlich M (2003) An exploratory analysis of extended grocery supply chain operations and home delivery. Integr Manuf Syst 14(8):652-663 
Braysy O, Gendreau M (2005) Vehicle routing problem with time windows, part ii: metaheuristics. Transp Sci 39(1):119-139

Carrabs F, Cerulli R, Sciomachen A (2014) Environmental sustainable fleet planning in B2C e-commerce Urban distribution networks". In: Dameri R, Rosenthal-Sabroux C (eds) Smart city. How to create public and economic value with high technology in urban space. Springer, Berlin

Carrabs F, Cerulli R, Speranza MG (2013) A branch-and-bound algorithm for the double travelling salesman problem with two stacks. Networks 61(1):58-75

Cattaruzza D, Feillet D, González-Feliu J (2015) Vehicle routing problems for city logistics. EURO J Transp Log, pp 1-29. doi:10.1007/ s13676-014-0074-0

Chao IM, Liou TS (2005) A new tabu search heuristic for the site dependent vehicle routing problem. In: Golden B, Raghavan S, Wasil $\mathrm{E}$ (eds) The next wave in computing, optimization, and decision technologies, vol 29. Springer, New York, pp 107-119

Chao IM, Golden BL, Wasil EA (1999) A computational study of a new heuristic for the site-dependet vehicle routing problem. INFOR 37:319-336

Confessore G, Galiano G, Stecca G (2008) An evolutionary algorithm for vehicle routing problem with real life constraints. In: The 41st CIRP conference on manufacturing systems, Tokyo

Cordeau J-F, Laporte G (2001) A tabu search algorithm for the site dependent vehicle routing problem with time windows. INFOR 39:292-298

Cordeau J-F, Desaulniers G, Desrosiers J, Solomon MM, Soumis F (2002) The vehicle routing problem. In: Toth P, Vigo D (eds) Volume 9 of SIAM monographs on discrete mathematics and applications 2002, Chap. 7, 157193. SIAM, Philidelphia

Crainic TG, Ricciardi N, Storchi G (2009) Models for evaluating and planning city logistics systems. Transp Sci 43(4):432-454

De Koster RBM (2002) The logistics behind the enter click. In: Klose A, Speranza MG, Van Wassenhove L (eds) Quantitative approaches to distribution logistics and supply chain management. Springer, Berlin, pp 131-148

Du TC, Li EY, Chou D (2005) Dynamic vehicle routing problem for online B2C delivery. Omega 33:33-45

Francis P, Smilowitz K (2006) Modeling techniques for periodic vehicle routing problems. Transp Res Part B 2006(40):872-884

Gosman C, Cornea T, Dobre C, Pop F, Castiglione A (2016) Putting the user in control of the intelligent transportation system. In: Lecture notes in computer science vol 9722, pp 231-246

Govindam K, Sarkis J, Chiappetta Jabbour CJ, Zhu K, Geng Y (2014) Eco-efficiency based green supply chain management: current status and opportunities. Eur J Oper Res 2014(233):293-298
Hsu C-I, Li H-C (2006) Optimal delivery service strategy for Internet shopping with time-dependent consumer demand. Transp Res Part E 42:473-497

Johnson ME, Whang S (2002) E-business and supply chain management: an overview and framework. Prod Oper Manag 11(4):413423

Kopfer W, Schönberger J, Kopfer H (2014) Reducing greenhouse gas emissions of a heterogeneous vehicle fleet. Flex Serv Manuf J 26(1):221-248

Lenstra JK, Rinnooy Kan AHG (1981) Complexity of vehicle routing and scheduling problems. Networks 11:221-227

Nag B, Golden B, Assad A (1988) Vehicle routing with site dependencies. Veh Routing methods Stud 16:149-159

Negru C, Mocanu M, Chiru C, Draghia A, Drobot R (2015) Cost efficient cloud-based service oriented architecture for water pollution prediction. In: Intelligent computer communication and processing (ICCP), pp 417-423

Petersen HL, Madsen OBG (2009) The double travelling salesman problem with multiple stacks: formulation and heuristic solution approaches. Eur J Oper Res 198(1):139-147

Pop F, Negru C, Ciolofan SN, Mocanu M, Cristea V (2016) Optimizing intelligent reduction techniques for big data. Stud Big Data 18:4970

Punakivi M, Saranen J (2001) Identifying the success factors in egrocery home delivery. Int J Retail Distrib Manag 29(4):156-163

Qureshi AG, Taniguchi E, Yamada T (2009) An exact solution approach for vehicle routing and scheduling problems with soft time windows. Transp Res E 45(6):960-977

Sarkis J, Zhu Q, Lai KH (2011) An organizational theoretic review of green supply chain management literature. Int J Prod Econ 130(1):1-15

Sniezek J, Bodin L, Levy L, Ball M (2001) Capacitated Arc routing problem with vehicle-site dependencies: the Philadelphia experience. In: Toth P, Vigo D (eds) The vehicle routing problem, pp 287-308

Simchi-Levi D, Wu DS, Shen ZJ (eds) (2004) Handbook of quantitative supply chain analysis: modeling in the E-business Era. Kluwer, Boston

Swaminathan J, Tayur SR (2003) Models for supply chains in Ebusiness. Manag Sci 49(10):1387-1406

Toth P, Vigo D (eds) (2015) Vehicle routing: problems, methods, and applications. Society for Industrial and Applied Mathematics, Pennsylvania

Yusuf I (2014) Solving multi-depot, heterogeneous, site dependent and asymmetric VRP using three steps heuristic. J Algorithms Optim $2: 28-42$ 